\title{
Temps et aspect dans le commentaire des illustrations d'articles de recherche en informatique
}

Pierre Labrosse

\section{OpenEdition \\ Journals}

Édition électronique

URL : http://journals.openedition.org/asp/2102

DOI : 10.4000/asp.2102

ISBN : 978-2-8218-0382-4

ISSN : 2108-6354

Éditeur

Groupe d'étude et de recherche en anglais de spécialité

Édition imprimée

Date de publication : 31 décembre 2000

Pagination : 155-186

ISSN : 1246-8185

Référence électronique

Pierre Labrosse, «Temps et aspect dans le commentaire des illustrations d'articles de recherche en informatique », ASp [En ligne], 27-30 | 2000, mis en ligne le 27 janvier 2011, consulté le 19 avril 2019. URL : http://journals.openedition.org/asp/2102; DOI : 10.4000/asp.2102

Ce document a été généré automatiquement le 19 avril 2019

Tous droits réservés 


\title{
Temps et aspect dans le commentaire des illustrations d'articles de recherche en informatique
}

\author{
Pierre Labrosse
}

\section{0 . Introduction}

1 Notre étude part de la confrontation de la position de L. Trimble avec les exemples trouvés dans notre corpus ${ }^{1}$. Il estime, en effet, que les auteurs d'articles de recherche choisissent le présent lorsqu'ils discutent d'une illustration :

\begin{abstract}
When writers discuss visual aids used in their discourse, the act of gathering the data for the illustration and the designing of the visual have already taken place. Thus, as in the case of description, the activities connected with the visual are, in the writer's mind, in the past. As a result, readers are told about gathering the data and designing the visual in the past tense. However, when writers discuss the visual itself and its relationship to the subject at hand, they choose the present tense. (1985: 125)
\end{abstract}

Il explique ensuite que la cause de ce changement de temps grammatical est rhétorique : les auteurs adoptent le point de vue du lecteur, qui se situe dans le hic et nunc de la lecture de leur article. Certes les proportions des différents temps utilisés dans notre corpus montrent que le présent est le temps le plus fréquemment utilisé dans ce commentaire ${ }^{2}$, loin devant le prétérit, qui n'offre pas d'écart significatif ici, et le present perfect dont l'emploi est significativement plus rare que ce qu'il devrait être.

Tableau 1. Commentaire d'une illustration (fonction B12) : les différents temps employés

\begin{tabular}{|l|l|l|l|l|}
\hline Temps & Tendance & Risque d'erreur & Nbre & $\%$ \\
\hline
\end{tabular}




\begin{tabular}{|l|l|l|l|l|}
\hline Prétérit & $\varnothing$ & $\varnothing$ & 32 & 12 \\
\hline Present perfect & - & $4 \%$ & 3 & 1 \\
\hline Présent & + & $1 \%$ & 232 & 86 \\
\hline Présent potentiel & $\varnothing$ & $\varnothing$ & 3 & 1 \\
\hline
\end{tabular}

3 Il n'en demeure pas moins que prétérit et present perfect apparaissent dans le commentaire des illustrations et que nous devons essayer d'en rendre compte à l'aide des outils que nous fournit la linguistique énonciative, dans laquelle nous incluons la théorie des opérations énonciatives de Culioli, la grammaire métaopérationnelle d'Adamczewski et Delmas, et la théorie de la réélaboration de Cotte.

\section{Respect de la valeur en langue des temps}

4 La première constatation que nous pouvons faire à partir de notre corpus est que l'emploi des temps grammaticaux que nous avons choisi d'étudier plus particulièrement parce qu'ils sont les plus fréquents dans notre corpus (le présent, le prétérit et le present perfect) est conforme à leur valeur en langue respective.

\subsection{Composantes de la valeur en langue du présent}

5 En ce qui concerne le présent, nous illustrerons successivement sa coïncidence avec le moment d'énonciation, sa réticence à exprimer une succession, sa souplesse d'emploi et l'impression qu'il donne de décrire la réalité telle qu'elle est.

\subsubsection{Coïncidence avec le moment d'énonciation}

P. Cotte analyse cette coïncidence en ces termes :

Une décomposition en traits sémantiques du sens du présent fait donc apparaître un trait inhérent, la coïncidence temporelle et personnelle, qui est l'invariant ou le signifié systématique propre de ce temps, et des traits contextuels contingents: emploi pragmatique ou épistémique, puis en fonction de ceux-ci, coïncidence totale, partielle, immédiate, médiate. Dans un énoncé au présent il y a donc répartition des tâches: au présent grammatical la coïncidence; au reste une propriété sémantique susceptible de produire l'effet final. (Cotte 1996 : 19)

Cette caractéristique permet de rendre compte de l'abondance de propositions au présent à cette fonction rhétorique: un commentaire dépend directement de l'énonciateur, l'auteur de l'article de recherche, qui exprime son jugement en T0. Cette fonction rhétorique de la proposition peut être évidemment renforcée par des marques explicites de commentaire, comme des adjectifs appréciatifs, des adverbes, des comparatifs et des superlatifs :

Ex. 1 B $12:\}$ Where the beam is perpendicular to the wall, the distance measurement is fairly accurate, but to either side of that point, the return is shorter than it should be. (IJRR : 287-290) 
Ex. 2 \{B12:\} Although $0^{3} \mathrm{MPCU}$ provides 0 significant reductions in the statespace search over the worst case, it is very poor compared to 0 MIACU. (JPDC : 738-741)

On remarque aussi des quantifieurs :

Ex. 3 \{B12:\} This of course produces many points along the $\mathrm{x}$ axis. (IEEESoft : 194-195) et même l'apparition de l'auxily
et du circonstant de moyen :

Ex. 4 \{B12:\} Only by including the error-free modules do you get a true picture, but even then the phenomenon of 0 falling error density persists (IEEESoft : 310-313).

9 On peut encore parler de coïncidence avec le moment d'énonciation dans cet énoncé générique, le pronom de $2^{\mathrm{e}}$ personne renvoyant au lecteur et à tout scientifique capable de comprendre le commentaire, car la validité des procès coïncide au moins en un point avec ce moment. Il en va de même lorsque le pronom de $1^{\mathrm{e}}$ personne inclut les auteurs, le lecteur et tout membre de la communauté scientifique :

Ex. 5 \{B12:\} If we move inward toward the center of the board, we see immediately that as we move from "ring" to "ring" the number of 0 cells attacked along 0 diagonals increases by two. (IBMJRD : 382-385)

\subsubsection{Atténuation de l'impression de succession}

La référence générique de ces sujets ne favorise pas particulièrement l'impression d'une succession des procès puisque leur validité se vérifie sur tous les moments, dont celui d'énonciation. Or $81 \%$ des pronoms personnels sujets pluriels dans les propositions commentant une illustration au présent partagent une telle référence.

11 Une autre caractéristique de ces propositions qui atténue l'impression de succession est le nombre d'anaphores de construction avec retour sur le nom tête lors de l'apparition de l'article défini the dans les syntagmes nominaux sujets ou objets. Non seulement ces anaphores ne s'appuient pas sur une succession textuelle de références à un même élément du réel, mais encore elles ne suivent pas le fil du texte comme c'est le cas dans the best regression (ex.10), anaphore de construction où le mouvement du discours apporte la détermination du superlatif best au nom regression : elles vont à contre-courant. Dans the phenomenon of falling error density (ex. 4) ou dans the number of cells attacked along diagonals (ex. 5), les repères (falling error density, cells attacked along diagonals) apportent leur détermination aux noms têtes de la construction en of, les repérés phenomenon et number.

12 Le même retour en arrière apparaitt avec les propositions subordonnées relatives, même tronquées, ces subordonnées déterminant leur antécédent :

Ex. 6 B B12:\} This example also shows how the multitasking facilities provided by 0 Sequent are used to create 0 multiple tasks, and how 0 objects can be passed to these processes (JOOP : 480-484).

Dans notre corpus, on peut compter plus d'anaphores de construction parmi les sujets de propositions au présent ( $49 \%$ ) qu'au prétérit $(31 \%)$, et une majorité $(76 \%)$ de ces 
anaphores reviennent sur le nom tête. Ce phénomène est encore plus frappant en ce qui concerne les COD, puisque l'article défini the s'y justifie par une anaphore de construction dans $95 \%$ des cas, une forte majorité ( $89 \%$ ) de ces anaphores opérant un retour sur le nom tête :

Ex. 7 \{B12:\} Similarly for the plot on the right, the crosses at the top of the room show that the infrared correctly finds the edges of the doorjamb again, while the sonar is completely blind to them. (IJRR : 393-397)

Ex. 8 \{B12:\} The vertical divisions show the point at which the queen in 0 Row 1 moves to a new column. (IBMJRD : 568-570)

Ex. 9 \{B12:\} (D8) This represents the knowledge that 0 sam worked on 0 programs 1 and 3, and 0 frank worked on 0 programs 2 and 3. (DKE : 422-424)

Notons que la détermination de ce dernier COD provient d'une subordonnée appositive (ou complétive) provenant de la proposition initiale that sam worked on programs 1 and 3, and frank worked on programs 2 and 3 is a (real) knowledge.

15 Ces anaphores suspendent donc l'écoulement du récit et contribuent à cette impression de statisme qui n'avait pas échappé à H. Chuquet :

En effet, ce qui apparaît encore plus nettement en anglais qu'en français, c'est l'existence de deux modes de construction différents de la succession. Au prétérit est associée une succession qui, se voyant assigner par rupture avec $T_{0}$ un point de départ situé dans le révolu, est orientée vers ce «but à atteindre » que constitue le repère-origine de sa construction. Avec le présent, au contraire, le repère de départ de la succession événementielle est donné, par le biais du décrochage fictif, comme contemporain d'une nouvelle origine énonciative, sans qu'il y ait mise en place d'une antériorité par rapport à $T_{0}$; la succession se construit donc à partir du premier événement de la séquence, chaque index d'événement servant de repère au suivant, indépendamment de toute progression vers le repère-origine absolu qu'est le moment de l'énonciation. C'est peut-être là une des raisons pour lesquelles le prétérit donne l'impression de créer la succession plus que ne le fait le présent ... même si ce n'est qu'une illusion. (1994 : 76-77)

\subsubsection{Souplesse d'emploi}

16 À la suite d'A. Culioli, elle a aussi souligné « la souplesse de ce marqueur » (9):

Les emplois du présent, ainsi que le montrent malgré elles les études qui tentent de les classer, s'inscrivent dans un continuum, tant il est vrai que cette forme offre un terrain privilégié aux distorsions, et constitue l'un des champs d'application les plus riches du concept de «déformabilité » défini par A. Culioli. (1990: 129-130; $1994: 8)$

17 Ceci entraîne la «superposition de valeurs multiples dans un même emploi » (9) ou des valeurs différentes dans des emplois différents :

Les formes de présent en contexte aoristique sont donc susceptibles de se voir attribuer toute une gamme de valeurs, qui ne relèvent pas toutes de la narration d'événements, et pour lesquelles la ligne de partage entre système aoristique et système de l'énonciation est loin d'être nette. Selon le type de procès concerné, la nature des arguments mis en relation, les marqueurs lexicaux apportant des déterminations spatio-temporelles ou aspectuelles, etc., ce qui va être plus ou moins filtré, c'est l'une ou l'autre des propriétés fondamentales de l'aoristique (rupture et/ou propriété “borné-fermé-compact» de l'intervalle associé au procès), ou la valeur d'indétermination sous toutes ses facettes, ou encore plusieurs de ces éléments à la fois. (Culioli $1990: 44)$ 
Cette souplesse est sans doute due à l'absence de marque sur la plupart des formes du présent, qui le rend plus perméable à l'influence de l'énoncé ou du contexte :

Cette prise en compte de toutes les parties constitutives de l'énoncé s'avère particulièrement nécessaire pour l'étude du présent, marqueur a priori « amorphe » et indéterminé. Agencements de marqueurs dans la chaîne linéaire, types de procès, déterminations aspectuelles : tels sont les paramètres à examiner afin de déterminer le statut narratif ou non du présent et de dégager les facteurs favorisant son apparition dans l'une et l'autre langue. (Culioli 1990 : 10)

Dans notre corpus, cette souplesse du présent se traduit tout d'abord par la grande variété des déterminants de syntagmes nominaux objets: les propositions à ce temps exemplifient dix des onze déterminants de ce syntagme, alors qu'on en rencontre seulement quatre dans celles au prétérit, deux dans celles au present perfect et un dans celles au présent potentiel.

Parmi les indéfinis, nous avons pu remarquer l'article $a(n)$ (a true picture - ex. 4), l'article zéro devant des discontinus pluriels $(\varnothing$ significant reductions in the state space search ex. 2) et le quantifieur many (many points - ex. 3). Nous pouvons ajouter l'article zéro devant un nom continu :

Ex. 10 \{B12:\} That is, while the best regression leads to a gain in 0 predictive confidence interval length of about 032 percent, the cross-validated model's intervals reduce $\varnothing$ length by about 026 percent. (ACMTDS : 460-464)

le déterminant one :

Ex. 11 \{B12:\} As it moves one step, it continues to attack $0 \mathrm{~N}-1$ cells on the main diagonal, but adds an additional two cells on the minor diagonal. (IBMJRD : 388-391)

et le quantifieur no:

Ex. 12 \{B12:\} Notice that we see no appreciable increase in 0 speed past 0 two processing elements. (JOOP : 549-551)

Parmi les définis, nous avons surtout observé l'article défini the (the edges of the doorjamb ex. 7, the point - ex. 8, the knowledge - ex. 9). Nous y ajouterons l'article zéro défini et le pronom it :

Ex. 13 \{B12:\} We arbitrarily select $\varnothing$ B, add it to $0 \mathrm{I}$, delete it from $0 \mathrm{~L}$, and delete $\varnothing \mathrm{C}$ from $0 \mathrm{~L}$ because it communicates with B. (JPDC : 519-522).

le déictique this :

Ex. 14 \{B12:\} We expand this node, again looking at all possible assignments of 0 module $\mathrm{C}$ and computing underestimates assuming that $0 \mathrm{~A}$ is assigned to 0 processor 3 (JPDC : $554-557$ )

et le génitif :

Ex. 15 \{B12:\} For 0 simple 3-order queries, the minimum response set size does not hinder the legitimate user's access to 0 information. (ACMTDS: 589-592)

Cette variété implique qu'aucun de ces déterminants n'offre d'écart significatif, tandis qu'on peut en remarquer parmi ceux des COD de verbes au prétérit ou au present perfect ${ }^{4}$. 
Cette souplesse s'exprime ensuite par une plus grande variété et une meilleure répartition de la référence des sujets entre les deux diathèses que celles que l'on observe au prétérit.

Tableau 2. Référents des sujets dans les propositions commentant une illustration au présent

\begin{tabular}{|l|l|l|l|l|}
\hline & \multicolumn{3}{|l|}{ Présent } & \multicolumn{2}{l|}{ Prétérit } \\
\hline Référent du sujet & \% voix active & $\begin{array}{l}\% \text { voix } \\
\text { passive }\end{array}$ & $\%$ voix active & $\begin{array}{l}\% \text { voix } \\
\text { passive }\end{array}$ \\
\hline auteur & $12 \%$ & $3 \%$ & $8 \%$ & $0 \%$ \\
\hline lecteur & $0 \%$ & $0 \%$ & $0 \%$ & $0 \%$ \\
\hline élément de l'expérience & $52 \%$ & $63 \%$ & $92 \%$ & $100 \%$ \\
\hline (élément de l') illustration & $22 \%$ & $7 \%$ & $0 \%$ & $0 \%$ \\
\hline élément de l'expérience & $11 \%$ & $27 \%$ & $0 \%$ & $0 \%$ \\
\hline \& de l'illustration & $1 \%$ & $0 \%$ & $0 \%$ & $0 \%$ \\
\hline anaphore & $100 \%$ & $100 \%$ & $100 \%$ & $100 \%$ \\
\hline Total & & & & \\
\hline
\end{tabular}

24 À l'exception des deux sujets référant l'un au lecteur, l'autre à un élément du contexte avant, tous les types de référents des sujets de propositions actives au présent se retrouvent à la voix passive, en particulier le pronom de 1e personne du pluriel qui réfère, entre autres, aux auteurs. Nous l'avons observé dans des propositions actives (we see, we move - ex. 5, we see - ex. 12, we select - ex. 13, we expand - ex. 14), mais il apparaît aussi dans des propositions passives :

Ex. $16\{B 12:\}$ Obviously, $I=\{B, E\}$, and thus we are left only with module A to assign independent of the assignments of any other modules. (JPDC: 944-947)

Les exemples 1 (the beam is perpendicular, the distance measurement is accurate, the return is shorter), 2 (MPCU provides / is poor) et 6 (the multitasking facilities provided by Sequent are used ) montrent que les sujets référant à un élément de l'expérience se rencontrent dans les propositions actives ou passives. On peut faire la même démonstration avec des sujets référant à (un élément de) l'illustration :

Ex. 17 B12:\} The right-hand figure shows the modified boundary after combining the near-infrared data with that from the sonar, with significantly more pronounced resolution of actual position. (IJRR : 475-480). Ex. 18 \{B12:\} In the Glass pattern shown in 0 Fig 3a, 0 short lines are detected at a small scale. (CVGIP : 568-569)

ou à un élément qui participe à la fois de l'expérience et de l'illustration : 
Ex. 19 B B12:\} The figure shows that the average branching factor diminishes by roughly two as each new queen is placed, until roughly half of the queens are placed. (IBMJRD : 344-348)

Ex. 20 \{B12:\} 0 Figure 5 also illustrates a feature that is found in several other figures. (JPDC : 720-722)

\section{l'illustration. Les propositions au présent peuvent ensuite dresser un compte rendu de} l'expérience de l'article :

Ex. 22 (1) B12: $\underline{W e}_{1}$ assign $0 \mathrm{~A}$, the first module on the list, to each processor and compute the MIACU cost for all three assignments of $0 \mathrm{~A}$. (2) B12: The node at 0 level $\underline{A}_{2}$ labeled 1 has the minimum underestimate of 115 and hence is chosen for expansion. (3) B12: Three child nodes are created, one for each possible assignment of $0 \mathrm{C}$, the next node in the module list. (4) B12: $\mathrm{We}_{1,3}$ observe that the underestimates computed for each of these assignments (all of which assume that $0 \underline{A}_{2,4}$ is assigned to 0 processor 1) are greater than the underestimate at the previous level for $0 \underline{A}_{4,5}$ assigned to 0 processor 3, which has an underestimate of 0 116. (5) B12: $\underline{\mathrm{We}}_{3}$ expand this node, again looking at all possible assignments of 0 module $\mathrm{C}$ and computing underestimates assuming that $0 \underline{A}_{5}$ is assigned to 0 processor 3 .

(6) B12: The remaining node at 0 level $\mathrm{A}$ is not expanded | B7: because there are 0 nodes at 0 level $C$ that have 0 lower underestimates. (JPDC: $540-561)^{5}$

28

On notera qu'ici les sujets référant aux auteurs, acteurs de l'expérience, apparaissent dans des propositions indépendantes ou principales, tandis que ceux référant à des éléments de l'expérience se trouvent dans les propositions subordonnées. Cependant, ce principe d'organisation ne se vérifie pas dans tous les passages au présent.

Une proposition au présent peut enfin dépeindre les conséquences de l'expérience sur l'illustration :

Ex. 23n(1) B12: The last column indicates the results of 0 regression on the synthetic of the unperturbed database as presented earlier. (2) B12: It can be noted that 0 perturbing the data has had little effect on the regression performance. (3) B12: Since with 0.25 perturbation, almost 03 of the perturbed values would be more than 025 percent away from the actual values, the regression methodology has succeeded even with 0 relatively large perturbation. (ACMTDS : 720-730)

Ce rôle discursif tient en partie à celui des énoncés suivants. Dans le deuxième, en effet, la principale ne fait que souligner la détermination que la périphrase du present perfect dans 
la subordonnée apporte à la situation présente. Dans le troisième, cette périphrase apporte la qualification de la proposition nominalisée (succeeded even with relatively large perturbation) à la situation d'énonciation et au thème de la proposition (the regression methodology) puisqu'il est défini. On peut penser que la qualification des deux propositions au present perfect s'applique à la fois à la situation d'énonciation et au thème de la proposition au présent (the last column), thème défini et situé au plan de l'énonciation par sa référence (élément de l'illustration), le temps du verbe (présent) et la catégorie de la proposition (indépendante).

31 Ainsi les propositions commentant une illustration au présent peuvent remplir trois rôles discursifs différents alors que celles au prétérit peuvent seulement décrire l'expérience de l'article ou parfois, mais rarement, référer à un passage antérieur du texte.

\subsubsection{Une impression de description de la réalité}

Dans les exemples que nous avons sélectionnés pour illustrer ces trois rôles discursifs, l'emploi d'un temps simple comme le présent permet aux auteurs de donner l'impression que la réalité se décrit elle-même. Certes cette illusion est mise à mal quand les sujets réfèrent aux auteurs ou lorsque des marques de commentaire apparaissent, mais l'ex. 21 est un assez bon exemple non pas à proprement parler d'un récit où «les événements semblent se raconter eux-mêmes " (Benveniste 1966: 241), mais d'une description où l'illustration puis ses différents éléments semblent arriver d'eux-mêmes à l'attention du lecteur. De même, nous pourrions paraphraser, à propos de l'ex. 22, la caractérisation du récit historique au prétérit donnée par $\mathrm{P}$. Cotte en disant que :

[...] le narrateur enregistre fidèlement, ou feint d'enregistrer, les événements d'un monde [...] objectif tels qu'ils [surviennent] et dans l'ordre où ils [le font], sans commentaire. Typiquement, le mode de l'énoncé est déclaratif: le narrateur enregistre et révèle ce qu'il [voit] à l'intention du narrataire, et la polarité est positive comme les réalités objectives rapportées. (1997b : 273)

Comme le montre notre corpus, cette caractérisation vaut pour les deux temps simples de l'anglais, le prétérit et le présent. Elle ne vaut bien entendu pas pour le present perfect, temps de discours.

\subsection{Composantes de la valeur en langue du present perfect}

\subsubsection{Une interruption du récit}

En effet, si le récit typique cherche à exprimer une succession d'événements, l'apparition d'un temps de discours dans cette succession est souvent ressentie comme une interruption de cette description du monde organisée selon l'écoulement du temps :

Dans ces deux passages ${ }^{6}$. les formes aspectuellement marquées s'opposent aux formes aoristiques du contexte environnant, repérant les procès comme étant accomplis par rapport au point de vue de he situé dans le récit, et venant rompre la succession événementielle. (Chuquet 1994 : 139) (c'est nous qui soulignons)

Nous pouvons avoir un doute sur cette caractéristique en observant que, dans notre corpus, l'article défini the déterminant des sujets n'offre pas d'écart significatif entre les propositions au present perfect et celles aux autres temps. En effet, cet article, marque d'anaphore, peut contribuer à la construction de successions événementielles : 
Ex. 24 (1) B12: From the first position, the robot 2 sees an area ${ }_{1}$ behind and to the left of the circular obstacle. (2) B12: From the second position, that area ${ }_{1,4}$ becomes occluded. (3) B12: After the robot ${ }_{2,3}$ moves forward, however, $\underline{\mathrm{it}}_{3}$ again sees the same area $\underline{4}_{4,5}$ behind the obstacle, only now $\underline{\mathrm{it}}_{3}$ sees $\underline{\mathrm{it}}_{5}$ from the right side. (IJRR: 627-634)

Sans oublier le rôle des autres marques anaphoriques, en particulier du pronom personnel it, l'article défini permet de comprendre que le même référent, le robot utilisé dans l'expérience de l'article, se déplace d'un point à un autre de la pièce et que ses deux positions doivent se comprendre en succession. Remarquons au passage que cet exemple n'infirme pas le fait que dans la grande majorité des propositions au présent, l'anaphore indiquée par cet article se justifie par la construction dans laquelle il figure.

Nous sommes donc en droit de penser que l'article défini the devrait apparaître significativement moins souvent dans les sujets des propositions au present perfect (comme c'est le cas dans les propositions mentionnant des recherches précédentes à ce temps, par exemple), ou bien qu'il devrait se justifier par une anaphore de construction dans la plupart des cas.

Étant donné le petit nombre d'occurrences des propositions au present perfect, notre analyse ne peut porter que sur le sujet de l'ex. 23, the regression methodology. Ce syntagme, qui figure dans le titre de l'article (The Use of Regression Methodology for the Compromise of Confidential Information in Statistical Databases) pourrait être considéré comme un bloc homogène. Même dans ce cas, il ne signale pas une succession à l'intérieur de l'extrait commentant une illustration dans lequel il figure. Notons qu'il permet souvent aux auteurs de cet article de considérer cette méthode en elle-même, sans la relier expressément à une mention textuelle précédente : using regression methodology de la ligne 123 suit the use of regression methodology (56-57). Nous préférons toutefois analyser ce syntagme comme un nom composé réélaborant une construction en of (the methodology of regression), mais où le mouvement du texte apporte la détermination du repère (regression ) au repéré (methodology).

En revanche, dans le COD de l'exemple suivant, le repère (the program of Fig. 1) apporte sa détermination au repéré (the processor flow graph) à contre-courant :

Ex. 25 \{B12:\} In Fig 12a, we have drawn the processor flow graph for the program of 0 Fig 1, using Stone's construction method. (JPDC : 931-934)

Par conséquent, le present perfect conserve toutes ses caractéristiques de temps de discours, malgré une absence d'écart significatif entre la proportion de sujets déterminés par l'article défini the à ce temps (33\%) et celle observée sur l'ensemble du corpus ( $49 \%$ ), ou malgré l'apparition de ce déterminant dans les COD des propositions au present perfect ici alors qu'il n'apparaît pas dans ceux des propositions mentionnant des recherches précédentes, ni dans ceux des propositions décrivant des appareils ou l'obtention des informations de l'illustration, ou de celles référant à la théorie à ce temps.

\subsubsection{L'intervention de l'énonciateur}

41 Une seconde caractéristique d'un temps de discours, et donc du present perfect, est la prise en charge par l'énonciateur de l'événement auquel réfère l'énoncé. P. Cotte pense que 
[...] la continuité énonciative est dans cette affaire essentielle, le fait que I saw him this morning coexiste avec I have seen him this morning est un signe certain que ce n'est pas le repérage référentiel qui décide de PPF [present perfect], même si traditionnellement la rupture référentielle avec $T_{0}$ exige, elle, l'emploi de ED [prétérit], mais la relation que l'énonciateur décide de coder entre le procès et son discours. (1987:116)

Dans son article de 1993, J. Chuquet précise un peu plus l'analyse :

Quel que soit le fonctionnement du procès, on aboutit donc avec le perfect à une stabilisation qualitative construite avec le $C_{0}$ comme repère, étant entendu que ce repère constitutif va lui-même se trouver repéré par rapport au système de coordonnées énonciatives et en particulier par rapport au point de vue d'un valideur qui, dans la plupart des cas, est identifié à l'énonciateur. (30-31)

On peut tout d'abord déceler cette intervention de l'énonciateur dans l'importance des sujets continus (risque d'erreur: $3 \%$ ) qui permettent de regrouper implicitement plusieurs éléments. Dans l'ex. 23, on peut dire que le sujet perturbing the data est la nominalisation d'un procès référant à une activité et qu'ainsi, à un certain niveau, il regroupe plusieurs actions identiques du point de vue notionnel. Il en va de même de the regression methodology, qui désigne une procédure, une démarche à suivre réunissant plusieurs procédés. On peut certes faire observer que ce rassemblement ne se montre pas, à la différence d'un discontinu pluriel marqué par la particule -s. C'est incontestable pour methodology; en revanche, la marque du gérondif dans perturbing pourrait bien signaler un regroupement d'actions?

Dans le même exemple, la prise en charge de l'énonciateur se remarque plus aisément sur le syntagme nominal objet (little effect), car le quantifieur little offre ici un écart très significatif (risque d'erreur : 1 pour cent millions). C'est une confirmation des remarques de P. Cotte : «[...] par contre, PPF [present perfect] doit, s'il est transitif, avoir un objet quantifié (* I've written / ? I've written letters / I've written two letters).» (1987: 123). Comme le signale H.Adamczewski dans l'introduction de son chapitre sur les quantifieurs, ces marqueurs sont des " traces d'opérations effectuées par l'énonciateur " (1982: 237) ; ceci se comprend assez facilement, puisqu'ils expriment la quantité que l'énonciateur juge adéquate au réel.

\subsubsection{Qualification de la situation présente par un procès passé}

L'exemple 23 nous a déjà donné l'occasion de montrer le lien que le present perfect établit entre la situation présente et le procès passé de la proposition nominalisée. Dans le deuxième énoncé, on peut dire que la principale (it can be noted) souligne le peu d'effet qu'a eu le brouillage des données lors de l'expérience (perturbing the data had little effect on the regression performance), inefficacité remise au premier plan de l'énonciation par le verbe recteur have au présent qui apparait pour former le present perfect (perturbing the data has had little effect on the regression performance). Nous avons aussi remarqué que les deux propositions au present perfect de cet exemple expliquent l'affirmation du premier énoncé au présent, explication qui forme un lien supplémentaire avec la situation d'énonciation.

De même, dans l'exemple 25, la proposition au present perfect décrit le résultat présent d'une action passée (we drew the processor flow graph for the program of Fig. 1). Même si le procès nominalisé qualifie aussi le référent du sujet défini we, car l'auteur est aussi le bénéficiaire du dessin de la figure 12a et participe de la situation d'énonciation, il apporte 
surtout sa détermination à la situation d'énonciation et au thème principal de l'énoncé, la figure 12a. On peut en effet analyser cet énoncé de la façon suivante ${ }^{8}$ (voir tableau 3).

Tableau 3. Analyse de l'énoncé de la figure 12a

\begin{tabular}{|l|l|l|}
\hline $\begin{array}{l}\text { In } \\
\text { Fig. 12a }\end{array}$ & we & $\begin{array}{l}\text { have drawn the processor flow graph for the program of Fig. 1, using } \\
\text { Stone's construction method. }\end{array}$ \\
\hline & $\begin{array}{l}\text { thème } \\
2\end{array}$ & propos 2 \\
\hline thème 1 & propos 1 \\
\hline
\end{tabular}

On remarquera que le circonstant de lieu antéposé (in Fig. 12a) fait partie de la situation d'énonciation et que le circonstant en fin d'énoncé (using Stone's construction method) se trouve du côté de la proposition au prétérit nominalisée en un participe passé. Ce circonstant indique le moyen d'une action révolue pour créer un objet (the processor flow graph for the program of Fig. 1) qui est visible au moment présent dans la figure 12a. Dans les autres propositions commentant une illustration au present perfect, les circonstants qui figurent dans la partie nominalisée de la périphrase sont liés à l'expérience de l'article et localisent ou qualifient des procès qui ont eu lieu lors de cette expérience : had little effect on the regression performance, has succeeded even with relatively large perturbation (ex. 23).

Malgré un nombre très réduit de propositions qui nous incite à la prudence, il semble que nous retrouvions ici une répartition des circonstants que nous pouvons observer par ailleurs dans les propositions décrivant l'obtention des informations de l'illustration au present perfect : les circonstants liés à la situation d'énonciation figurent dans une position privilégiée, en tête de proposition, ce qui leur confère le rang de thème gouvernant le reste de la proposition'. C'est cette référence issue de leur expérience que les auteurs veulent mettre en avant. L'analyse de J. Chuquet souligne bien le lien du repère constitutif avec le système de coordonnées énonciatives, en particulier l'énonciateur valideur de l'assertion. Elle n'est pas sans rappeler les remarques d'E. Benveniste sur le parfait :

Le parfait établit un lien vivant entre l'événement passé et le présent où son évocation trouve place. C'est le temps de celui qui relate les faits en témoin, en participant ; c'est donc aussi le temps que choisira quiconque veut faire retentir jusqu'à nous l'événement rapporté et le rattacher à notre présent. (1966:244)

En revanche, les circonstants liés à l'expérience figurent en fin d'énoncé, dans la partie nominalisée de la périphrase. De façon iconique, le verbe recteur have se situe au milieu de ces deux types de circonstants car il traduit le lien que crée l'énonciateur entre le passé de l'expérience et le présent de l'illustration.

\subsection{Composantes de la valeur en langue du prétérit}

\subsubsection{L'antériorité à $T_{0}$}

Le prétérit, quant à lui, semble se focaliser sur le passé de l'expérience, comme le montrent la référence de ses sujets et celle de ses circonstants. Tous les sujets des propositions commentant une illustration à ce temps renvoient à l'expérience, qu'il 
s'agisse d'éléments de l'expérience (94\%) ou des auteurs (6\%), qui en sont les initiateurs ; le pourcentage des sujets référant à un élément de l'expérience présente d'ailleurs un écart très significatif (risque d'erreur: 1 pour dix mille). Parmi ceux référant à des éléments de l'expérience, nous remarquons, outre sam, frank de l'ex. $9^{10}$ :

Ex. 26 B $12:\}$ First, the output unit was inhibited at most times between to and $\mathrm{t} 1$; this was most pronounced when the activity threshold was high (B-11aN: Fig $4 \mathrm{~d}$ and e) because then the inhibitory units inactive at $\mathrm{t} 1$ greatly outnumbered the excitatory units active at that time. (NeuComp: 512-518)

Parmi ceux référant aux auteurs, nous pouvons citer :

Ex. 27\{B12:\} This is an example of the tradeoffs we made between 0 run-time efficiency and 0 information hiding and 0 other abstraction issues. (JOOP : 267-270)

51 Notons aussi que l'absence de sujets référant à un élément de l'illustration ou à un élément de l'illustration et de l'expérience est significative, voire très significative dans le premier cas (risque d'erreur : 1 pour dix mille).

On observera aussi que la plupart des COD renvoient à des éléments de l'expérience : $60 \%$ si l'on adopte une définition stricte (the excitatory units active at that time - ex. 26), 80 \% si l'on accepte la combinaison avec une autre référence :

Ex. 28 \{B12:\} The original boundary is shown in the left-hand picture with the new boundary overlaid on it at the locations where the programmable proximity sensor marked the edges. (IJRR : 471-475)

Les bords ont été marqués par le capteur lors de l'expérience et ils apparaissent dans la figure 8 de l'article que cet énoncé commente.

Ces tendances concordent avec la forte proportion de propositions incluant un circonstant référant uniquement à l'expérience (78\%). On aura remarqué les circonstants de lieu (on programs 1 and 3 et on programs 2 and 3) de l'ex. 9 et les circonstants de temps et de lieu de l'ex. 26 : at most times, between to and t1, (at the moment) when the activity threshold was high. On peut aussi rencontrer des circonstants de moyen et de manière :

Ex. 29 \{B12:\} Although this is not an exhaustive description of how the model is constructed, it should give the reader an idea of how it was implemented through an object-oriented approach to 0 systems design. (JOOP : 300-304)

Le commentaire d'une illustration montre même que ces circonstants sont plus fréquents dans les propositions passives au prétérit, dans lesquelles un circonstant figure toujours, ce qui n'est pas le cas des propositions actives. Puisque l'agent n'est jamais ressorti, il semble bien que la construction passive permette ici de focaliser l'attention du lecteur sur les circonstances du procès. Nous en voulons pour preuve les exemples 26 et 29 , où les circonstants (at most times between to and t1, through an object-oriented approach to systems design) occupent tout l'espace textuel après le verbe.

Notons aussi que le reste des circonstants dans les propositions commentant une illustration au prétérit réfèrent à la fois aux circonstances de l'expérience et à celles de l'illustration, comme dans cet énoncé : 
Ex. 30 \{B12:\} The crosses mark where the infrared detects 0 corners or, specifically, where 0 infrared readings of 30 transitioned to anything else. (IJRR : 380-383) 'enonciation est la délimitation du procès par des bornes fermées :

En l'absence de définition de $<\mathrm{Cp}>$ [complémentaire de $\mathrm{p}$ ], la validation de $\langle\mathrm{p}>$ est présentée comme : “ $\langle\mathrm{p}>$, un point c'est tout », sans qu'il soit possible d'établir de lien aspectuel ou même notionnel avec le contexte.

Figure 1. Bornes fermées du prétérit (Fryd 1995 : 201)

\begin{tabular}{|c|c|}
\hline R. & \\
\hline 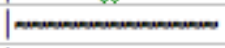 & {$\left[T_{0}\right.$} \\
\hline L & Cp \\
\hline
\end{tabular}

Bien qu'elles ne présentent pas ici d'écart significatif, les proportions des différents fonctionnements des sujets de verbes au prétérit ne sont pas inintéressantes: on remarque une majorité de discontinus singuliers $(53 \%)$; viennent ensuite les discontinus pluriels ( $28 \%$ ) et les continus (19\%). Il semble bien que ces sujets renvoient de préférence à des référents délimités, en particulier des délimités uniques. Ceci s'observe plus particulièrement parmi les sujets déterminés par l'article zéro défini, qui réfèrent à des entités (sam, frank - ex. 9) ou à des variables :

Ex. 31 \{B12:\} As $\varnothing$ alpha dropped further, 0 discrimination of 0 repeated cycles fell off. (NeuComp : 361-363)

Ex. 32 \{B12:\} $\varnothing$ D was already included in the set of 0 independent nodes, but $\varnothing \mathrm{C}$ was not. (JPDC : 939-941)

61 En ce qui concerne les COD, on constate plus de syntagmes nominaux discontinus pluriels (the excitatory units active at that time - ex. 26, the edges - ex. 28) que de discontinus singuliers :

Ex. 33 \{B12:\} You can perform no significant curve fitting on these data, so we used a bar graph to show the average error density found in all modules grouped by 0 size (B11aN: 0 Figure 3). (IEEESoft : 195-199) 
En revanche, aucun COD continu n'apparaît, ce qui plaide en faveur de l'association d'un procès au prétérit avec des référents délimités.

De plus, l'autre déterminant des COD discontinus singuliers prend une importance bien plus grande que dans les propositions aux autres temps (risque d'erreur : $2 \%$ ) :

Ex. 34 \{B12:\} The scatter graph of 0 error density versus 0 size for 0 modules that had at least one error shows that an artifact of 0 discrete data contributes to the impression of 0 falling error density. (IEEESoft : 305-310)

64 Or ce déterminant renforce la singularisation de l'article indéfini $a(n)$ :

L'article a singularise un continu; le déterminant one singularise de même un discontinu ; ex. : I soon saw that it had one fatal drawback / All I could do was to offer you an opinion upon one minor point. La singularisation est quantitative (un seul) et qualitative (un certain bien précis).... Comme l'article a marque aussi un singulier qualitatif et quantitatif (cf. a week or two ago / an hour's discourse), on considérera one comme son renforcement. (Cotte 1996 : 215)

65 Comme le référent de ce COD désigne un élément de l'expérience, nous retrouvons l'orientation du prétérit vers la réalité passée de l'expérience et son association avec un référent délimité unique.

\subsubsection{La construction d'une succession}

66 Notre paraphrase de P. Cotte à propos de la caractérisation du récit historique rappelle que le narrateur emploie le régime énonciatif de l'histoire pour « [enregistrer], ou [ feindre] d'enregistrer, les événements d'un monde révolu objectif tels qu'ils sont survenus et dans l'ordre où ils l'ont fait, sans commentaire.» (1997b : 273). Dans la théorie des opérations énonciatives, la caractérisation des procès au prétérit comme autant d'intervalles bornés fermés sans possibilité de chevauchement va de pair avec la construction d'une chronologie entre plusieurs procès au prétérit ${ }^{11}$. Cette caractéristique fait du prétérit le temps prototypique du récit.

67 Or dans les propositions commentant une illustration à ce temps, l'article défini the déterminant un sujet ou un COD se justifie plus souvent par une anaphore textuelle que dans les propositions aux autres temps (risque d'erreur : $1 \%$ et $4 \%$ respectivement). Il faut toutefois examiner plus précisément ces anaphores.

8 Certes le COD de l'exexemple 28 (the edges) reprend the two edges of the opening quelques lignes auparavant dans l'article d'IJRR (1.467). Mais d'une part la source de l'anaphore se situe dans un circonstant, non dans un sujet ou un COD qui référerait à un participant du procès et, d'autre part, cette anaphore ne renforce pas l'unité textuelle d'un passage uniquement consacré au commentaire de l'illustration : sa source se situe dans un énoncé décrivant la démarche expérimentale ; de plus, un énoncé référant à la figure 8 s'intercale entre cette source et l'apparition de l'anaphore.

Un phénomène similaire se produit pour les sujets :

Ex. 35 (1) B12: It can be seen that the inhibitory units had two effects. (2) B12: First, the output unit was inhibited at most times between to and $\mathrm{t} 1$; this was most pronounced when the activity threshold was high (B11aN: Fig $4 \mathrm{~d}$ and e) because then the inhibitory units inactive at $\mathrm{t} 1$ greatly outnumbered the excitatory units active at that time. (NeuComp: 510-518) 
Dans ce commentaire de la figure 4 de l'article de Neural Computation, les sources des anaphores sont extérieures au passage :

- the inhibitory units reprend all inhibitory units (1.508), dans un énoncé décrivant la démarche expérimentale ;

- the output unit rappelle the output unit's input activity (1.495), dans la légende d'une illustration ;

- the activity threshold réfère à the activity threshold, alpha (1.502), dans une phrase décrivant l'obtention des informations de l'illustration.

Seul le pronom déictique this reprend la proposition qui le précède, contribuant ainsi à l'unité textuelle du passage.

71 Si dans les syntagmes nominaux sujets ou objets de verbes au prétérit, l'anaphore de l'article défini the s'appuie sur l'écoulement du texte, il nous faut cependant convenir qu'elle ne contribue pas à renforcer l'unité textuelle formée par un ensemble de propositions à ce temps, alors qu'on peut observer ce phénomène dans des passages mentionnant des recherches précédentes :

Ex. 36 B2a: 0 ABEL (0 Patil, 1981), a program for diagnosing 0 acid-base and electrolyte disturbances, maintained a description of a patient's illness at $\varnothing$ five levels of 0 detail. B2a: The least detailed level represented 0 associational knowledge and the more detailed levels were used for 0 model-based reasoning. B2a: However, rather than choosing when to solve a problem by using 0 associational reasoning and when to use 0 model-based reasoning, $0 \mathrm{ABEL}$ always reasoned about the patient at every level of 0 detail. (AAI : 457-469)

Il se pourrait aussi que cette anaphore ne contribue pas particulièrement au renforcement d'unités textuelles en raison du petit nombre de passages au prétérit ou d'extraits au prétérit et au présent commentant une illustration. L'exemple 35 montre que les auteurs emploient le prétérit plutôt pour décrire des événements de l'expérience sans les organiser en une succession.

\subsubsection{Un lien implicite avec $T_{0}$}

73 L'antériorité des faits de l'expérience par rapport à $T_{0}$, que nous avons étudiée dans la section 1.3.1, n'empêche pas un lien implicite de ces faits avec le moment d'énonciation, même s'il n'est dû qu'à l'expression de commentaires qui rappellent que l'énonciateur se situe à ce moment quand il utilise ce temps du passé.

74 Nous avons ainsi pu remarquer un adjectif exprimant une évaluation (high - ex. 26), un comparatif (further - ex. 31), un superlatif (most pronounced - ex. 26), un adverbe (greatly). Nous pouvons ajouter une marque explicite de comparaison, accompagnée d'ailleurs d'un autre superlatif :

Ex. 37 \{B12:\} The cross-validated $0 \mathrm{R} 2$ was 0.454 , as compared with the best value of .54. (ACMTDS : 458-460)

et un quantifieur :

Ex. $38\{B 12:\}$ When 0 alpha $=0.999$, too few units were selected and 0 discrimination of even the original interval was poor. (NeuComp : 356-358).

exemple où l'on observe aussi les adverbes intensifs too et even, ainsi que l'adjectif poor. 
Les marques de commentaire ne manquent donc pas dans les propositions commentant une illustration au prétérit, et il n'est pas surprenant de constater qu'elles apparaissent plus souvent au premier plan de l'énonciation, puisque l'énonciateur se trouve à ce plan lorsqu'il exprime un commentaire : elles figurent dans $86 \%$ des indépendantes et $71 \%$ des principales, mais seulement dans $39 \%$ des subordonnées, qui se situent à l'arrièreplan de l'énonciation.

Il semble plus difficile en revanche de maintenir que les faits évoqués puissent être toujours vrais au moment présent, comme dans He was here a moment ago, où il n'est pas explicitement exclu qu'il puisse en réalité être encore ici. Il est toutefois possible de souligner que la recherche scientifique postule que les événements de l'expérience décrits dans l'article puissent être reproduits à tout moment, dont celui d'énonciation. C'est en tous cas ce que les auteurs prétendent, aidés en cela par la caution de leurs pairs du comité de lecture. Par conséquent, un lien implicite se tisse encore entre le révolu de l'expérience et la réalité présente, sans oublier la nécessité de considérer intellectuellement deux moments ensemble pour ensuite les séparer et placer l'un dans le présent, l'autre dans le révolu - opération valable pour tout verbe au prétérit.

\subsubsection{Une impression de description de la réalité telle qu'elle était}

Les marques de commentaire que nous venons d'évoquer ne semblent pas corroborer l'impression de description de la réalité que nous avons pu mettre en avant pour le présent, l'autre temps simple de l'anglais. Toutefois, dans les propositions à ce temps, nous avons noté que cette impression était également mise à mal par des marques de commentaire. Observons cependant que l'impression de commentaire est moins forte avec un verbe à un temps simple qu'avec une forme périphrastique, qui ajouterait une marque de commentaire sur l'un des deux constituants essentiels de la proposition, celui qui est lié au sujet par la conjugaison. Dans les propositions commentant une illustration au prétérit de notre corpus, nous constatons une absence d'écarts significatifs entre les déterminants indéfinis ou définis du sujet et ceux des sujets de propositions aux autres temps. Nous pouvons expliquer ce phénomène par une certaine conformité au réel.

On constate en effet que les éléments de l'expérience sont introduits dans l'article à l'aide de syntagmes nominaux déterminés par l'article zéro indéfini, syntagmes qualifiés par le repère d'une construction en of ou par une subordonnée relative : $\varnothing$ infrared readings of 30 (ex. 30), $\varnothing$ discrimination of repeated cycles (ex. 31), $\varnothing$ discrimination of even the original interval (ex. 38), $\varnothing$ modules that had at least one error (ex. 34).

D'autre part, notre étude de l'exemple 35 a montré que les sujets déterminés par l'article défini the reprennent des éléments déjà présentés dans l'article. Il en va de même pour ceux déterminés par un article zéro défini : dans l'exexemple 31, alpha réfère au seuil d'activité dont il est question à la ligne 291 (activity threshold alpha), et les variables $D$ et $C$ de l'ex. 32 désignent des modules mentionnés dans l'énoncé précédent (module $C$, module $D$ ). Même si ces sources de reprise sont elles-mêmes déterminées par un article zéro défini, tout ce qui importe ici est que les sujets déterminés par cet article dans les propositions au prétérit sont présentés comme des reprises et qu'ils constituent bel et bien des reprises.

Notre étude des articles définis the dans la section 1.1.3 ne permet toutefois pas de soutenir que dans les passages ou extraits où figurent des verbes au prétérit, un même élément est d'abord introduit par un syntagme nominal indéfini puis repris par un 
syntagme nominal défini. Nous pouvons simplement comparer les proportions de ces déterminations avec celles que l'on observe dans les propositions mentionnant des recherches précédentes au prétérit, où les trois-quarts des sujets dont définis parce qu'ils sont déjà connus de la communauté scientifique. Ici nous observons une répartition plus équilibrée de la détermination, plus conforme à la réalité de la présentation d'une expérience nouvelle, où les éléments de cette expérience doivent d'abord être présentés au lecteur qui les découvre avant de lui demander de se les remémorer dans des énoncés ultérieurs, même si l'énoncé qui comprend l'anaphore et celui où se situe sa source ne commentent pas tous deux une illustration.

81 L'étude des propositions commentant une illustration montre donc que les proportions de différents constituants des propositions corroborent des composantes de la valeur en langue de chaque temps grammatical. Cette valeur en langue implique par définition une invariance qui tendrait à gommer la spécificité de l'emploi des temps pour une fonction rhétorique donnée. Mais notre corpus montre que des caractéristiques propres au commentaire d'une illustration apparaissent aussi au travers de certains constituants.

\section{Influence de la fonction rhétorique}

\subsection{Un prétexte pour exposer la recherche de l'article}

82 Il n'est peut-être pas surprenant, en effet, que les auteurs mettent plus l'accent sur la description de leur expérience dans les propositions au prétérit, puisque cette expérience a eu lieu dans une période révolue. Ainsi nous avons pu montrer dans la section 1.3.1 que tous les sujets de ces propositions renvoient à l'expérience, et un grand nombre de leurs circonstants réfèrent uniquement à cette expérience (88 \%).

Cette orientation vers l'expérience est un peu plus surprenante dans les propositions au present perfect, car ce temps peut établir un lien entre l'expérience et la situation d'énonciation, dont l'illustration fait partie. Dans l'exemple 25, il est permis de dire que le pronom personnel sujet (we have drawn) réfère au sujet hors de l'acte, alors que le sujet de la proposition initiale (we drew the processor flow graph for the program of Fig. 1) renvoie au sujet dans l'acte de tracer un organigramme lors de l'expérience ${ }^{12}$. Une telle solution ne peut être maintenue pour des sujets comme perturbing the data ou the regression methodology (ex. 23) qui renvoient à des éléments de l'expérience.

Cette description de l'expérience est tout à fait inattendue dans les propositions au présent, surtout lorsqu'on sait que dans la description de l'obtention des informations de l'illustration, les sujets des propositions à ce temps renvoient plus fréquemment à l'illustration ou des éléments de l'illustration (risque d'erreur : $1 \%$ ). Ici nous avons certes pu dégager une plus grande variété de références parmi les sujets de verbes au présent (section 1.1.3) ; mais le tableau 2 montre aussi que les sujets référant à des éléments de l'expérience sont la majorité, tant dans les propositions actives que passives. En fait, si l'on additionne les références à un élément de l'expérience, celles à un élément de l'expérience et de l'illustration, et celles aux auteurs, nous aboutissons à $78 \%$ des sujets de verbes au présent. En adoptant les mêmes critères, tous les sujets des propositions au prétérit ou au present perfect renvoient à l'expérience.

Dans les propositions au présent, cette majorité de sujets référant à l'expérience est aussi renforcée par une très forte majorité de COD $(95 \%)$ et une forte majorité de circonstants 
(74\%) partageant la même référence. Les passages au présent deviennent donc des comptes rendus de l'expérience de l'article, comme le prouvent l'exemple 22 ou le passage suivant :

Ex. 39 (1) B12: Note that, in general, $\underline{\text { a queen }}_{1}$ in a corner attacks $0 \mathrm{~N}-1$ cells along a diagonal. (2) B12: If you move the queen ${ }_{1,2}$ in the corner along the periphery of the board, $\underline{i t}_{2}$ continues to attack $0 \mathrm{~N}-1$ cells, because as the $\underline{\text { queen }}_{2}$ moves from 0 cell to 0 cell, the length of one diagonal shortens and the other diagonal lengthens by the same amount. (3) B12: Hence, in Figure 2, $\underline{w e}_{3}$ show that each cell on the periphery attacks seven cells along 0 diagonals.

(4) B12: If $\underline{w e}_{3}$ move inward toward the center of the board, $\underline{w e}_{3}$ see immediately that as $\underline{w e}_{3}$ move from 0 " ring ò » to 0 « ring " the number of 0 cells attacked along 0 diagonals increases by two. (5) B12: (D8) For example, $\underline{a}$ queen $_{4}$ in the corner can be moved one step along a diagonal toward the board center. (6) B12: As $\underline{\mathrm{it}}_{4}$ moves one step, $\underline{\mathrm{it}}_{4}$ continues to attack $0 \mathrm{~N}-1$ cells on the main diagonal, but adds an additional two cells on the minor diagonal. (7) B12: (D8) Thus, the board may be viewed as composed of 0 concentric rings as shown in 0 Figure 2, with each ring closer to the center attacking two more cells than its nearest outer neighboring ring. (IBMJRD: 370-395)

En fait, les auteurs commentent une illustration en tant que représentation de leur expérience, ce qui les amène naturellement à parler surtout de leur expérience dans des propositions dont nous pensions qu'elles devraient plutôt décrire l'illustration en tant que telle. Il n'est pas étonnant que le prétérit et le présent se prêtent facilement à ce commentaire (notons que ce sont les temps qui offrent ici le plus de propositions) car ils permettent à l'auteur de prétendre qu'il décrit la réalité telle qu'elle est. Le present perfect convient beaucoup moins bien à cette description de l'expérience servant de commentaire à l'illustration, bien qu'il puisse établir un lien entre l'expérience révolue et l'illustration actuelle; il est donc significativement moins bien représenté que dans les autres fonctions rhétoriques de notre corpus. D'une certaine façon, l'illustration n'est qu'un prétexte à la description de l'expérience de l'article, orientation qui impose des choix sur les temps grammaticaux, sur la référence des syntagmes nominaux sujets ou objets, et sur celle des circonstants.

\subsection{Un commentaire}

Cette première caractéristique de la fonction rhétorique que nous étudions ne doit pas pour autant faire oublier qu'elle apporte un commentaire, même s'il s'agit souvent d'un commentaire de l'expérience de l'article telle qu'elle est exposée dans les illustrations.

On remarque donc plusieurs quantifieurs dans les sujets de verbes au prétérit, dont few dans l'exemple 38. Nous relevons aussi le quantifieur no dans le composé nothing et all :

Ex. $40\{$ B13\}: For the particular scan which produced this plot, only three LEDs were used (one was temporarily inoperative), B12: so the reading of 30 at the point (168 85) shows that at the maximum range of the system, nothing was detected when all three LEDs were fired. (IJRR : 351-357)

Notons que les quantifieurs few et no sont significativement plus fréquents que dans les propositions aux autres temps (risque d'erreur : $2 \%$ ). Nous savons par ailleurs ( $c f$. section 
1.1.2) que le quantifieur little apparaît plus souvent dans les COD des propositions au present perfect (ex. 23 ; risque d'erreur : 1 pour cent millions).

Dans la section 1.3.4, nous avons détaillé les fréquentes marques de commentaire qui apparaissent dans les propositions au prétérit, qui sont ici légèrement plus nombreuses (56\%) que dans celles au présent (51\%). Dans les propositions que nous avons citées, l'auteur commente des faits ancrés dans l'expérience, donc révolus, mais qui sont illustrés dans une figure ou un tableau présents en $T_{0}$. Si le prétérit met en avant le récit de l'expérience, nous savons que l'énonciateur reste au moment d'énonciation lorsqu'il expose ces faits passés. C'est de cette position qu'il peut commenter les faits illustrés par une figure, et nous ne devons pas nous étonner qu'il use de cette possibilité dans des propositions dont la fonction rhétorique est précisément celle de commentaire, d'autant plus que ces marques de commentaire n'altèrent pas l'objectivité des informations scientifiques car elles sont données dans l'illustration et non dans le texte.

On peut aussi penser que la fonction de commentaire n'est pas étrangère à la proportion de verbes d'état tant dans les propositions au prétérit que dans celles au présent. Dans les premières, si les sujets référant aux auteurs se conjuguent prototypiquement avec des verbes d'action ou d'activité à la voix active (we used a bar graph - ex. 33, we made tradeoffs - ex. 27), on constate que $55 \%$ des sujets référant à des éléments de l'expérience se conjuguent avec des verbes d'état ou de localisation, ce qui représente $38 \%$ des propositions actives: the activity threshold was high (ex. 26), the cross-validated $R 2$ was .454 (ex. 37), [modules] had one error (ex. 34), the inhibitory units had two effects (ex. 35). Cette proportion est bien différente des $36 \%$ que ces verbes représentent dans les propositions actives décrivant l'obtention des informations de l'illustration au prétérit.

La proportion de verbes d'état ou de localisation dans les propositions commentant une illustration au présent est légèrement plus importante (43\%). Là encore ces verbes apportent la qualification du procès à des sujets référant à des éléments de l'expérience : the beam is perpendicular, the distance measurement is fairly accurate, the return is shorter (ex. 1), [MPCU] is very poor (ex. 2), the sonar is completely blind (ex. 7), the underestimates ... are greater (ex. 22), there is a set of three numbers (ex. 21), the node at level A labeled 1 has the minimum underestimate of 115, [processor 3] has an underestimate of 116 (ex. 22). Comme au prétérit, les sujets référant aux auteurs s'associent à des verbes d'action $(96 \%)$ ou d'activité (4\%): les auteurs conduisent l'expérience (we select, add, delete, and delete ex. 13, we move from "ring" to "ring» - ex. 5, we expand this node - ex. 14) ou la démonstration (we show that... - ex. 39), ou bien dirigent l'attention du lecteur tout en percevant un fait intéressant (we see that... - ex. 5, we see no appreciable increase - ex. 12).

\subsection{Un éclatement de la fonction rhétorique spécifique de relation entre texte et illustration}

La nature de la fonction rhétorique devrait aussi nous permettre d'expliquer la moindre proportion de verbes au present perfect et l'importance de ceux au présent, alors que ces proportions sont inversées dans la description de l'obtention des informations de l'illustration, bien qu'il s'agisse toujours d'une fonction rattachée à l'illustration. 


\subsubsection{Proportions des temps dans la description de l'obtention des informations de l'illustration (B13/B13p)} l'article lui-même, mais elle s'intéresse à l'obtention des informations lors de la recherche plutôt qu'à l'illustration. En d'autres termes, elle établit un lien entre deux périodes, les résultats de la recherche ayant une incidence sur la représentation actuelle. Cette recherche est non seulement objectivement révolue, mais encore présentée comme révolue dans des propositions au prétérit :

Ex. 41 \{B13:\} To obtain the results plotted in 0 Fig 5, we held the P:C ratio constant (at 5) and looked at the performance of 0 MIACU for the number of processors equal to 3,4 , and 5 . (JPDC : 714-717)

Ex. 42 \{B13:\} For this specific example we used a lower cutoff of 0 ten and an upper cutoff of 0 size 100 , below which 0 triangles were recursively subdivided and not pushed back into the stack. (JOOP: 598-602)

Ex. 43 \{B13:\} A population of 250 pacemakers was used to store an interval of $2 \mathrm{sec}$, with activity thresholds varied from alpha $=0.999$ to 0.0. (NeuComp : 347-350)

Ce temps est donc plus fréquemment utilisé à cette fonction rhétorique (risque d'erreur : 1 pour un milliard). On ne sera pas surpris de constater que les sujets des verbes à ce temps réfèrent plus souvent à des éléments de l'expérience (risque d'erreur : $1 \%$ ).

Le present perfect est alors nécessaire pour expliciter les conséquences de cette recherche sur la période où se situe l'illustration, le moment d'énonciation, ce qui se traduit aussi par un nombre significativement plus important de propositions à ce temps (risque d'erreur : 1 pour dix mille) :

Ex. 44 \{B13p\}: In each [graph] the $\mathrm{x}$ axis has been scaled to the stored interval and the $y$ axis to the number of pacemakers selected to encode that interval. (NeuComp : 272-275)

Ex. 45 \{B13p:\} 0 Figure 5 has been scaled according to the total number of cells selected, so to make the output threshold clear a line has been drawn at beta $=70$ in each graph, which is the average number of 0 units chosen for each interval. (NeuComp : 547-553)

Notons que dans ces deux exemples, la référence à l'illustration se situe dans le sujet (the $x$ axis, figure 5, a line), référence qui n'est pas loin de présenter un écart significatif (risque d'erreur: $6 \%$ ), et parfois dans le circonstant de lieu antéposé (in each [graph]). Les circonstants situés dans la partie nominalisée de la périphrase réfèrent à l'expérience (to the stored interval, to the number of pacemakers selected to encode that interval, according to the total number of cells selected, at beta $=70$ ).

Puisque l'illustration n'est pas le centre d'intérêt principal de ces énoncés, le présent y est relativement moins utilisé qu'ici (risque d'erreur : $2 \%$ ). Les sujets des verbes à ce temps renvoient plus fréquemment à des illustrations ou des éléments de l'illustration qu'à des éléments de l'expérience (risque d'erreur : $1 \%$ ) :

Ex. 46 \{B13p:\} The earlier examples of three different views of the room are translated and scaled appropriately, then overlaid two at a time so as not to become too cluttered. (IJRR : 620-623) 


\title{
2.3.2. Proportions des temps dans le commentaire d'une illustration
}

\subsubsection{Présence de l'illustration au moment d'énonciation}

\begin{abstract}
au moment d'énonciation, même si le récit de son expérience en constitue l'essentiel. Il ne fait pas le récit de sa recherche en tant qu'expérience passée, ce qui explique que la proportion de propositions au prétérit n'offre pas d'écart significatif. Par conséquent, il a beaucoup moins besoin de construire un lien entre cette recherche passée et l'illustration présente, d'où la moindre proportion de propositions au present perfect (risque d'erreur : $4 \%$ ). Comme le montre l'ex. 22 , il présente sa recherche comme commentaire de l'illustration, donc en coïncidence avec cette illustration : il s'agit d'une mise en parallèle, d'une sorte de diptyque illustration / recherche, l'illustration résumant graphiquement la recherche, la description de la recherche éclairant l'illustration par les explications qu'elle lui apporte. Les propositions au présent sont donc plus nombreuses ici (risque d'erreur : $1 \%$ ).
\end{abstract}

\subsubsection{Le développement de l'illustration dans le texte}

100 Un autre élément peut expliquer l'importance des propositions aux temps simples par rapport à celles au present perfect: la condensation opérée dans l'illustration. Cette opération nous a été suggérée par l'analyse de T. Miller :

To summarize then, the visuals in the Science article highlight the argument how the scientists arrived at their views. Comparison and contrast dominates the visuals, which also are very hierarchical within the cells of each point of contrast. Notice too how much text the visuals dominate. In fact, one could argue that the purpose of main text is to clarify the [visuals] and provide implications for the visuals. In other words, one could argue that the visuals dominate the text rather than the other way around. In fact, as Huckin has demonstrated, specialists in a discipline move straight to the visuals, ignoring the main text. (1996:31)

En d'autres termes, le texte développe l'illustration. Si nous mettons ce rapport en parallèle avec les mouvements de condensation/déploiement dans les extraits mentionnant des recherches précédentes, nous pouvons penser que le texte doit déployer, expliciter l'illustration dans des propositions aux temps simples :

Ex. 47 Condensation : B2a: But 0 counterexamples have been given that show that the human visual system does not use 0 gray-level blurring in its simplest form.

Expansion 1A : B2a: In [19] a number of 0 counterexamples are presented. Expansion $2 \mathrm{~A}: \mathrm{B} 2 \mathrm{a}$ : One is an image consisting of a Glass pattern with 0 black dots on a gray background superimposed on a Glass pattern with 0 white dots on a gray background. B2a: The patterns are superimposed in such a way that the black and white dots cancel if the input is low-pass filtered. B2a: Nevertheless the perception of 0 flow remains.

Expansion 1B : B2a: Another counterexample was presented in [22] using the method of 0 Carlson et al [3].

Expansion $2 \mathrm{~B}$ : $\mathrm{B} 2 \mathrm{a}$ : This counterexample involved a Glass pattern with a gray background where each white dot is surrounded by an appropriately chosen little black « moat » (B11aN: 0 Fig 4). (CVGIP: 146-164)

Comme une illustration est présente au moment d'énonciation de l'article, l'emploi du présent est favorisé par rapport à celui du prétérit, qui présenterait le commentaire et la 
recherche de l'article comme décalés dans le passé. D'autre part, il ne s'agit pas de revenir sur l'illustration en tant que moment antérieur du texte, ni même la plupart du temps, de la présenter comme le résultat de recherches passées : elle est le centre d'intérêt à ce moment de l'article, elle est autonome, et le texte est simplement posé en parallèle spatialement et temporellement. Ceci explique la quasi-absence de propositions au present perfect, temps de commentaire ${ }^{13}$ lorsqu'il revient sur un énoncé du texte, mais qui n'a pas ici à revenir sur l'illustration. On pourrait s'étonner qu'un temps de discours, où l'énonciateur signale expressément sa présence au travers du verbe recteur have, ne soit pas plus employé ici. Le paradoxe n'est qu'apparent et réside dans le mot " commentaire", qui se limite ici à poser un texte en parallèle à une illustration, sans s'interdire d'autres marques de commentaire.

\subsubsection{Conclusion : une utilisation temporelle des temps}

Malgré cette seconde hypothèse à propos de l'utilisation plus fréquente du présent, nous devons convenir que dans le commentaire d'une illustration comme dans la description de l'obtention des informations de l'illustration, l'auteur utilise les temps grammaticaux plutôt selon leur expressivité temporelle que rhétorique. C'est parce que l'objet à commenter, l'illustration, est présent au moment d'écriture (et de lecture) de l'article que les auteurs choisissent le présent pour s'exprimer, même si ce commentaire consiste en un récit de l'expérience, qui est alors posée en coïncidence avec l'illustration. C'est parce que l'objet qui a permis l'obtention des informations données dans l'illustration, l'expérience, est révolu qu'ils optent pour le prétérit pour la raconter. Notre analyse rejoint donc celle de L. Trimble ${ }^{14}$. sauf que nous pensons tout d'abord qu'il s'agit d'un emploi des temps selon leur ancrage temporel et, ensuite que nous sommes en présence de deux, voire plusieurs, fonctions rhétoriques et non d'une seule, comme il l'affirme :

Level $\mathrm{C}$ is made up of the specific rhetorical functions that are found most commonly in written EST discourse: description, definition, classification, instructions, and visual-verbal relationships between a visual aid and its accompanying text. Most commonly the discourse at this level is presented either in groups of closely related paragraphs or in single paragraphs. (1985: 11 - c'est nous qui soulignons)

Plus loin dans son livre, il apporte quelques précisions sur la rhétorique de ces relations entre le visuel et le verbal :

By visual-verbal we mean the relationships between visual aids such as drawings, schematics, graphs, tables, charts --any illustrative material- and a piece of text. The function of visual aids is to add information to that given by the discourse; as a result, we find visual aids only in conjunction with other rhetorical features. (ibid. : 102)

105 Outre le fait que L. Trimble a une conception du rapport entre l'illustration et le texte inverse à celle de T. Miller, nous remarquons que cette fonction en recouvre trois parmi celles distinguées par « l'école de Washington $»^{15}$ dans les années 1970 : la référence à une illustration, le commentaire d'une illustration et la description de l'obtention des informations de l'illustration. De toute évidence, une telle réunion ne permettrait pas d'observer une inversion des proportions de present perfects et de présents entre deux fonctions rhétoriques qu'elle ne différencie pas. 


\section{L'absence de formes en be + ing}

\subsection{Un paradoxe}

Nous avons expliqué dans la section 2.3.2.2 pourquoi la faible proportion de propositions au present perfect ne constitue pas vraiment un paradoxe. Un autre paradoxe semble résider dans le fait qu'aucune forme en be+ing ne soit utilisée pour exprimer ce commentaire d'une illustration. En effet, U. Dubos ferait sans doute remarquer que le présent n'établit pas de distance entre l'énoncé et la référence, comme dans cette légende d'un tableau: The English attack Quebec ${ }^{16}$. Or la distance semble nécessaire au commentaire, et la forme en be+ing construit cette distance en revenant sur une première conjugaison :

$\mathrm{Be}+$ ing construit deux fois le même procès ; cette répétition est responsable d'une propriété importante. La première conjugaison attribue un temps au procès, l'inscrit dans le monde et l'actualise. Au moment d'énonciation -lors de la seconde conjugaison - cette actualisation préconstruite est acquise, même si elle n'a pas été dite effectivement. L'énonciation ne peut donc pas fonder le procès, mais seulement en prendre acte, y retourner, comme à une réalité extérieure objective. (Cotte 1997a : 98-99)

Cette forme semble donc toute désignée pour commenter une illustration. Cela serait la conséquence logique (ou naturelle) des propriétés d'extériorité et de séparation dont P. Cotte souligne l'importance :

Chaque fois [dans chaque énoncé à l'imperfectif cité auparavant] il est référé à une perception objectivée dont l'énonciateur se distancie pour la commenter, l'évaluer ou la mettre en question. (102 - c'est nous qui soulignons)

Cette extériorité et cette séparation sont la source d'une anaphore, car l'énonciateur revient sur une réalité préexistante :

Le procès installé dans le monde est indépendant de l'énonciateur. [...] La dimension anaphorique de be +ing a été soulignée récemment et elle est le pendant de l'extériorité discutée en a ${ }^{17} \backslash$. Elle explique que de nombreux énoncés en be +ing aient une composante expressive et modale: la reprise se prête à l'ajout d'un commentaire, qui peut être explicite (cf. At last I'm seeing Venice) ou implicite (cf. Am I hearing what you are saying ?). (104)

La périphrase, forme analytique, permet ainsi une analyse et une interprétation tandis que la forme simple inscrit naturellement le procès dans une description ou une succession.

\subsection{La distance objective entre le texte et l'illustration}

110 Cependant, dans les articles de recherche, même si la forme simple n'établit pas de distance entre le texte et l'illustration, une telle distance existe objectivement car texte et illustration sont deux entités différentes. Il n'est donc pas nécessaire de créer une distance entre elles avec une forme périphrastique. Deux pôles coexistent : l'illustration présente des informations (condensation), et le texte les explique (développement). $\mathrm{Si}$ l'on suit l'analyse de T. Miller, qui lui-même s'inspire de T. Huckin ${ }^{18}$, le texte est second par rapport à l'illustration. Il pense même que « les illustrations contrôlent tout le texte dans l'article de recherche scientifique $»^{19}$. Par conséquent, des commentaires peuvent apparaitre dans ce texte parce que les informations scientifiques objectives figurent dans 
l'illustration : ils ne risquent pas d'altérer la précision de ces informations. C'est la raison pour laquelle nous avons pu remarquer que la majorité des propositions au présent ou au prétérit comprennent des marques de commentaire. Il en va de même de celles au present perfect (67\%), sans prendre en compte le verbe recteur have évidemment.

111 En revanche, en ce qui concerne la description de l'obtention des informations de l'illustration, le lecteur ne peut pas retrouver dans l'illustration les étapes de l'expérience décrites par le récit au prétérit : l'illustration n'en présente que l'étape finale, le résultat. Quand l'auteur se permet d'insérer des commentaires dans son exposé, ils altèrent l'objectivité de la seule source d'information sur les étapes de l'expérience.

112 Ici, le statut du texte de l'article par rapport à l'illustration permet l'apparition de marques de commentaire avec des formes simples. De plus, l'absence de formes be + ing et la faible fréquence des present perfects évitent à l'auteur de souligner son intervention. Il sauvegarde ainsi un semblant d'objectivité, vite démenti toutefois par l'apparition d'autres traces de son intervention.

\subsection{L'illustration comme instantané de l'expérience}

113 Remarquons enfin qu'une illustration présente un instantané de l'expérience : il s'agit de quelque chose de stable, que l'on peut assimiler à un état, l'état des lieux à un moment donné de l'expérience. Elle ne décrit pas l'évolution de l'expérience, bien que la succession des illustrations de l'article traduise cette évolution, comme les images fixes d'une pellicule projettent un mouvement quand elles défilent devant l'appareil de projection. En commentant une seule illustration à la fois, nul n'est besoin d'interrompre un mouvement pour en indiquer le but, comme le ferait une forme en be + ing.

Si le procès est imperfectif, et intercepté, on perçoit seulement des sous-actions, alternées ou non, différentes à chaque instant, dont on infère l'identité du procès général. Ce saut jusqu'à l'être succède logiquement à la perception du phénomène sensible. Une fois qu'il est effectué, l'identité abstraite (walk, take a train) est reversée aux instants pendant lesquels dure le procès.

Le procès imperfectif est ainsi « reconstruit » aux deux sens du terme : son identité est découverte par une inférence (parfois minimale) et elle est répétée dans la chaîne de ses instants. (Cotte 1997a :105-106)

114 Dans le commentaire d'une illustration, deux constituants sont simplement posés l'un à côté de l'autre : le texte explicite l'illustration par le commentaire qu'il exprime, mais il est nullement nécessaire de recourir aux formes en be +ing pour créer une séparation entre les deux, car elle existe déjà, ou pour intercepter une évolution quelconque, car l'illustration est statique.

\section{Conclusion}

Le commentaire d'une illustration n'implique donc pas un travail rhétorique important de la part de l'auteur : il n'a besoin ni de se distancer de la cible de son commentaire ni de la stabiliser. Il pose simplement ce commentaire en coïncidence avec sa cible, coïncidence qui l'amène " naturellement » à utiliser le présent puisque l'illustration est bien visible au moment d'énonciation. Cet emploi «naturel » du présent a cependant des répercussions rhétoriques dont l'auteur ne peut que se féliciter : comme le thème de ce commentaire est souvent lié à l'expérience exposée dans l'article, cette expérience est alors présentée 
comme valable en $T_{0}$, et même au-delà, lorsque le sujet inclut le lecteur et tout membre de la communauté scientifique. Ainsi un emploi du présent qui se justifie tout d'abord par des considérations temporelles finit par se charger d'implications rhétoriques, non parce que l'auteur prend le point de vue du lecteur, mais parce que le présent, en actualisant l'expérience passée, élargit sa validité.

116 Il nous faut reconnaître cependant que ce travail énonciatif relativement limité explique sans doute le moindre intérêt de cette fonction rhétorique en ce qui concerne l'emploi des temps. Certes nous avons pu dire que l'illustration n'y est qu'un prétexte pour exposer la recherche de l'article, ce qui explique l'importance de la référence des sujets, des COD et des circonstants à l'expérience. De plus, les fortes proportions des marques de commentaire et de verbes d'état dans le texte ne sont bien entendu pas étrangères à la fonction rhétorique de ce texte. Mais on n'y rencontre pas de témoins d'une réorganisation du réel par le texte aussi forte que le mouvement de condensation / déploiement illustré dans l'exemple 47.

117 Ce moindre intérêt est peut-être renforcé par le nombre relativement peu élevé de propositions commentant des illustrations dans les articles de recherche de notre corpus, bien qu'elles ne soient pas les plus mal représentées. Ce déficit est ensuite aggravé par le déséquilibre entre les différents temps grammaticaux. La conjugaison de ces deux facteurs est indubitablement une source de difficultés pour une analyse statistique des phénomènes présents dans ces propositions. Il est en effet difficile de dégager des écarts significatifs à partir de si petits nombres, surtout lorsqu'on doit ensuite subdiviser les phénomènes: propositions au prétérit, articles définis dans ces propositions, article défini the, article défini the justifié par une anaphore de construction, article défini the justifié par une anaphore de construction revenant sur le nom tête. On aboutit ici à un conflit entre l'approfondissement de l'analyse et les exigences de la statistique.

118 La solution évidente serait d'élargir le corpus ; mais on dépasse rapidement les limites du traitement des données par une personne dans une durée fixée, malgré l'outil informatique. Nous avons donc souvent dû nous contenter de comparaisons de pourcentages, sans pouvoir dégager d'écart significatif. Ceci n'implique ni que la statistique soit inutile, ni que ces mêmes pourcentages ne puissent devenir significatifs dans des études ultérieures sur des corpus plus conformes aux exigences de cette science. Ces comparaisons de pourcentages sans écart significatif permettent simplement d'avancer des hypothèses en prenant un risque d'erreur bien plus grand que celui généralement accepté.

119 Malgré ces réserves d'ordre rhétorique ou statistique, l'analyse linguistique nous a permis de montrer que les auteurs d'articles de recherche en informatique sinon exploitent, du moins respectent, la valeur en langue des temps que nous avons étudiés, à commencer par leur ancrage temporel. Nous avons aussi pu observer l'expression de cette valeur en langue dans le fonctionnement, la référence et la détermination des syntagmes nominaux sujets ou objets, dans la référence et la position des circonstants, et même dans les marques de commentaire des propositions au prétérit ou dans la variété des rôles discursifs de celles au présent.

Cette valeur en langue est en fait un faisceau de caractéristiques temporelles, aspectuelles et modales que l'on peut formaliser en un système topologique, mais sans oublier que l'énonciateur peut choisir de valoriser une ou certaines caractéristiques plutôt que de les exprimer toutes ensemble. Notre corpus montre que les composantes temporelles, 
aspectuelles et modales d'une forme verbale particulière ressortent plus ou moins suivant le contexte dans lequel l'auteur les plonge. P. Cotte choisit l'image de l'anémone de mer, plante fixée au rocher mais dont la fleur bouge au gré du courant, pour figurer l'expression changeante de l'invariant de la valeur en langue au gré du texte. Nous espérons que notre étude aura montré que cette plante s'adapte fort bien aux courants des langues pour spécialistes d'autres disciplines.

\section{BIBLIOGRAPHIE}

\section{Composition du corpus}

Connelly, Steven \& Azriel Rosenfeld. 1990. « A pyramid algorithm for fast curve extraction ». Computer Vision, Graphics and Image Processing 49 : 332-345. (abrév. CVGIP)

Factor, Robert M. \& William B. Smith. 1988. « A discipline for improving software productivity ». AT\&T Technical Journal 67/4 : 2-9. (abrév. ATTTJ)

Flynn, A. M. 1988. « Combining sonar and infrared sensors for mobile robot navigation ». The International Journal of Robotics Research 7/6 : 5-15. (abrév. IJRR)

Harrison, Warren. 1989. «PDSS A programmer's decision support system ». Data \& Knowledge Engineering, $4:$ 115-123. (abrév. DKE)

Koton, Phyllis A. 1989. «A method for improving the efficiency of model-based reasoning systems ». Applied Artificial Intelligence 3/2-3 : 273 [357]-281 [365]. (abrév. AAI)

Miall, Christopher. 1989. « The storage of time intervals using oscillating neurons ». Neural Computation 1/3 : 359-371. (abrév. NeuComp)

Palley, Michael A. \& Jeffrey S. Simonoff. 1987. « The use of regression methodology for the compromise of confidential information in statistical databases ». ACM Transactions on Database System 12/4 : 593-608. (abrév. ACMTDS)

Sinclair, J. B. 1987. « Efficient computation of optimal assignments for distributed tasks ». Journal of Parallel and Distributed Computing $4: 342-362$. (abrév. JPDC)

Stepoway, Stephen L. \& Michael G. Christiansen. 1989. « Object-oriented fractal modeling on a shared-memory MIMD Machine ». Journal of Object-Oriented Programming 2/1 : 20-25. (abrév. JOOP)

Stone, Harold S. \& Janice M. Stone. 1987. « Efficient search techniques. An empirical study of the N-Queens Problem ». IBM Journal of Research and Development 31/4 : 464-474. (abrév. IBMJRD)

Winters, Jack H. \& Christopher Rose. 1989. « Minimum distance automata in parallel networks for optimum classification ». Neural Networks $2: 127-132$. (abrév. NeuNet)

Withrow, Carol. 1990. « Error density and Size in Ada software ». IEEE Software : 26-30. (abrév. IEEESoft)

\section{Références bibliographiques}

Adamczewski, Henri. 1993. Grammaire linguistique de l'anglais, $4^{\mathrm{e}}$ édition. Paris : Armand Colin. Benveniste, Émile. 1966. Problèmes de linguistique générale, tome 1. Paris : Gallimard. 
Berkenkotter, Carol \& T. N. Huckin. 1995. Genre Knowledge in Disciplinary Communication: Cognition/ Culture/Power. Hillsdale, New Jersey Hove, G.-B. : Lawrence Erlbaum Ass, Inc.

Chuquet, Hélène. 1994. Linguistique contrastive et traduction ( $N^{\circ}$ spécial) - Le présent de narration en anglais et en français. Gap \& Paris : Ophrys.

Chuquet, Jean. 1993. "Perfect anglais et opérations de validation : quelques repères ». In DanonBoileau L. \& Jean-Louis Duchet (dir.), Opérations énonciatives et interprétation de l'énoncé. Mélanges offerts à Janine Bouscaren. Paris: Ophrys, 27-45.

Cotte, Pierre. 1987. « Réflexions sur l'emploi des temps du passé en français et en anglais à la lumière de deux évolutions récentes du système verbal de l'anglais ». Contrastes 14-45, 89-161.

Cotte, Pierre. 1996. L'explication grammaticale de textes anglais. Paris : Presses Universitaires de France.

Cotte, Pierre. 1997. Grammaire linguistique. Paris : Didier Éruditions/ CNED.

Culioli, Antoine. 1990. Pour une linguistique de l'énonciation, Tome 1. Gap : Ophrys.

Descles, Jean-Pierre \& Zlatka Guentchéva. 1980. « Construction formelle de la catégorie grammaticale de l'aspect (Essai) ». In David \& Martin (dir.) : 195-237.

Fryd, Marc. 1995. « La périphrase /HAVE + PP/ en anglais contemporain : opérations énonciatives et construction de l'aspect accompli », Thèse de doctorat en linguistique théorique et formelle, Université de Paris 7.

Heslot, Jeanne. 1983. « Récit et commentaire dans un article scientifique ». D.R.L.A.V. 29 : 133-154.

Labrosse, Pierre. 1995. «Critères de sélection d'un corpus : exemple de l'anglais de

l'informatique ». Cahiers de l'APLIUT, 15/1:31-45.

Labrosse, Pierre. 1997. « Quelle rhétorique pour les articles scientifiques ?». ASp 15-18 : 185-206.

Labrosse, Pierre. 1999. "L'aspect en anglais de l'informatique », Thèse de doctorat en linguistique, Université de Paris-Sorbonne Paris IV.

Miller, Tom. 1995. «Cultures and genres », Conférence au XVII ${ }^{e}$ congrès de l'APLIUT, Belfort, 8-10 juin 1995.

Miller, Tom. 1996. «Visual perception: The role of visuals in academic articles and popularizations ». Cahiers de l'APLIUT 15/3, 23-35.

Selinker, Larry, Louis Trimble \& Robert Vroman. 1972. Working Papers in Scientific and Technical English. Office of Engineering Research and Department of Humanistic-Social Studies, College of Engineering, University of Washington. Texte dactylographié : 94-122.

Todd-Trimble, Mary \& Louis Trimble. 1982. « Rhetorical-grammatical features of scientific and technical texts as a major factor in written ESP communication ». In Hoedt J. et al. (dir.), Pragmatics and LSP, Proceedings of the 3rd European symposium on LSP. Copenhagen, 17-19 August 1981, 199-216.

Trimble, Louis. 1985. English for Science and Technology. A discourse approach. Cambridge :

Cambridge University Press. 


\section{NOTES}

1. Les références des douze articles qui constituent ce corpus sont données en annexe. Nous en avons exposé les critères de sélection dans notre article de 1995.

2. Bien que le terme de « commentaire » soit chargé de nombreuses connotations en linguistique énonciative, nous avons préféré le conserver car il nous semble être le plus fidèle à la définition que L. Selinker et al. donnent de cette fonction rhétorique dans leur article de 1972 : interpretation of the information of the graph with direct reference to the experiments (112). On trouvera une définition des fonctions rhétoriques que nous utilisons dans notre article de 1997.

3. Nous avons marqué l'article zéro par 0 ou $\varnothing$ dans nos exemples.

4. Cf. les sections 1.2.2 et 1.3.2.

5. Les verbes des propositions indépendantes ou principales sont en gras; les verbes des subordonnées sont en italique. Les anaphores et leurs sources sont soulignées et numérotées lorsqu'il y en a plus d'une. Nous avons numéroté chaque phrase pour faciliter les références.

6. Voici les deux exemples donnés par H. Chuquet: (107) - You mean they knew who you were? They asked you? Nerve enough for anything. - Of course not. - He has crumpled the bit of paper, transparent with grease, he wipes his fingers on it as best he can, and now he winds down his window. $(\mathrm{N}$. Gordimer, The Conservationist, p. 137)

(108) He asks where the chiefs herdsman is. Without moving, but grimacing as if she strains to hear, she makes an assenting noise and then answers. He repeats what she has said, to be sure, and she repeats the assenting noise, long and reassuring, like the grunting sigh of a satisfied sleeper. Her gaze steers his back in the direction she has indicated.. (N. Gordimer, The Conservationist, p. 10)

7. Nous reprenons ici l'hypothèse de P. Cotte (séminaire de DEA du 3 juin 1999) selon laquelle la marque -ing du gérondif (-ung en vieil anglais) est la nominalisation du présent général. Elle a remplacé la marque -ende du participe présent, nominalisation du présent ponctuel, parce que, ne pouvant plus rien ajouter à la répétition du procès indiquée par le présent général, elle devenait disponible pour le participe présent. De plus, les deux nominalisations étaient sémantiquement très proches : elles disaient (et disent encore) la répétition du procès sur une classe d'instants.

8. Nous nous inspirons ici de l'analyse que P. Cotte donne des phrases comme «Sur la fenêtre, des pierres luisaient au soleil ».

9. Cf. Labrosse $1999: 821-824$.

10. sam et frank (notons l'absence de majuscules) ne renvoient pas exactement à des personnes, mais à des entités « programmeur » associées à différents programmes.

11. «Les propriétés topologiques du système aoristique sont distinctes du système fondamental de l'énonciation: chaque "état de choses » borné sera représenté par un intervalle fermé (non nécessairement ponctuel); deux fermés seront ou disjoints ou emboîtés ou confondus; tout empiétement d'intervalles fermés bornés est exclu parce que non significatif, alors qu'un tel chevauchement nous a conduit aux intersections d'intervalles considérés dans le premier système de repérage [le système fondamental de l'énonciation, centré sur l'origine $\left.T_{0}\right]$; [...] La structure du système aoristique est alors directement compatible avec une chronologie marquée par des dates par exemple. » (Desclés \& Guentcheva $1980: 221$ )

12. "Quand le sujet est une réalité complexe, comme un être vivant, l'action où il s'engage le dédouble ; d'un côté est le référent dont l'identité transcende les différents actes qu'il accomplit pendant son existence; de l'autre est l'agent se mêlant à la matière dans l'acte particulier. » (Cotte 1997a : 109) 
13. Dans son article de 1983, J. Heslot reprend l'analyse de Weinrich : «Pour l'anglais, Weinrich retient le prétérit comme temps du récit, le présent et le Present Perfect comme temps du commentaire.» (144).

14. Cf. la citation donnée dans l'introduction (1985:125).

15. Cette appellation réfère à J. Lackstrom, L. Selinker, L. Trimble et R. Bley-Vroman, membres de l'équipe à laquelle M. Todd-Trimble et L. Trimble font allusion en 1982: "Our first research team at the University of Washington developed the term 'non-temporal use of tense' several years ago in an attempt to identify a particular set of rhetorical-grammatical relationships in which it appeared that the choice of verb tense was made by the writer on some basis other than that of time; that is, time was not the primary factor in the choice of tense. " (203-204)

16. Remarque faite lors de la soutenance de thèse de M. Petit, le 17 décembre 1993, à l'institut d'anglais Charles V - Université Paris 7.

17. La citation précédente est issue du paragraphe a), consacré à l'extériorité et la séparation.

18. T. Miller pense en particulier au passage suivant : «All of our consultants looked for visual aids as soon as they had finished reading the title and abstract, and all showed great facility in interpreting them .» (Berkenkotter \& Huckin 1995 : 37).

19. Conférence au XVII e congrès de l'APLIUT (Belfort, 8-10 juin 1995).

\section{RÉSUMÉS}

Selon L. Trimble (1985 : 125), l'auteur emploie le présent pour discuter de l'illustration et de sa relation avec le sujet abordé dans l'article. On constate cependant, dans les articles de recherche en informatique, qu'il utilise aussi le prétérit et le present perfect. Nous montrons tout d'abord que les conditions de l'emploi de ces trois temps respectent leur valeur en langue et étudions ensuite l'influence de la fonction rhétorique de commentaire d'une illustration sur ces constituants. L'utilisation des temps qui y est faite diffère donc de celle que l'on peut observer dans la description de l'obtention des informations de l'illustration. Nous terminons avec un paradoxe : les formes en be + ing sont absentes alors qu'elles soulignent typiquement une distance entre l'énoncé et la réalité, écart favorable au commentaire.

According to L. Trimble (1985: 125), an author uses the simple present to interpret an illustration and relate it to the discussion at hand in the article. It is to be observed, however, that he also uses the simple past and the present perfect to this end in computer science research articles. In this article we show that the conditions for use of these three tenses are consistent with their language value. We then study the influence of the rhetorical function. Relating an Illustration to the Discussion on these constituents: it not only serves as a commentary on an illustration but it also offers an opportunity to explain the experiment described in the article itself. Therefore the use of tenses here differs from that observed in sentences presenting information on data gathering in an illustration. We end with a paradox: be + ing forms are not used here although they typically underline the distance between an utterance and the reality it refers to, a gap which tends to give rise to comments. 
INDEX

Mots-clés : aspect, fonction rhétorique, illustration, temps

Keywords : aspect, illustration, rhetorical function, tense

\section{AUTEUR}

\section{PIERRE LABROSSE}

Pierre Labrosse, ancien élève de l'École normale supérieure de Saint-Cloud, agrégé d'anglais, a soutenu une thèse de doctorat sur l'aspect en anglais de l'informatique en décembre 1999. Après avoir été chargé d'une maîtrise de conférences à l'Université Paris IX-Dauphine, il est actuellement maître de conférences à l'Université Paris IV-Sorbonne. Il y enseigne la linguistique en LCE et l'anglais de spécialité en LEA. Pierre.Labrosse@paris-sorbonne.fr 